\title{
Efficient MR Image Reconstruction for Compressed MR Imaging
}

\author{
Junzhou Huang, Shaoting Zhang, and Dimitris Metaxas \\ Division of Computer and Information Sciences, \\ Rutgers University, \\ NJ, USA 08854
}

\begin{abstract}
In this paper, we propose an efficient algorithm for MR image reconstruction. The algorithm minimizes a linear combination of three terms corresponding to a least square data fitting, total variation (TV) and $L 1$ norm regularization. This has been shown to be very powerful for the MR image reconstruction. First, we decompose the original problem into $L 1$ and TV norm regularization subproblems respectively. Then, these two subproblems are efficiently solved by existing techniques. Finally, the reconstructed image is obtained from the weighted average of solutions from two subproblems in an iterative framework. We compare the proposed algorithm with previous methods in term of the reconstruction accuracy and computation complexity. Numerous experiments demonstrate the superior performance of the proposed algorithm for compressed MR image reconstruction.
\end{abstract}

\section{Introduction}

Magnetic Resonance (MR) Imaging has been widely used in medical diagnosis because of its non-invasive manner and excellent depiction of soft tissue changes. Recent developments in compressive sensing theory [1] 2] show that it is possible to accurately reconstruct the Magnetic Resonance (MR) images from highly undersampled K-space data and therefore significantly reduce the scanning duration.

Suppose $x$ is a MR image and $R$ is a partial Fourier transform, the sampling measurement $b$ of $x$ in $\mathrm{K}$-space is defined as $b=R x$. The compressed MR image reconstruction problem is to reconstruct $x$ giving the measurement $b$ and the sampling maxtrix $R$. Motivated by the compressive sensing theory, Lustig et al. 3] proposed their pioneering work for the MR image reconstruction. Their method can effectively reconstruct MR images with only $20 \%$ sampling. The improved results were obtained by having both a wavelet transform and a discrete gradient in the objective, which is formulated as follows:

$$
\hat{x}=\arg \min _{x}\left\{\frac{1}{2}\|R x-b\|^{2}+\alpha\|x\|_{T V}+\beta\|\Phi x\|_{1}\right\}
$$

where $\alpha$ and $\beta$ are two positive parameters, $b$ is the undersampled measurements of $\mathrm{K}$-space data, $R$ is a partial Fourier transform and $\Phi$ is a wavelet transform. It is based on the fact that the piecewise smooth MR images of organs can be sparsely represented by the wavelet basis and should have small total variations. The TV

T. Jiang et al. (Eds.): MICCAI 2010, Part I, LNCS 6361, pp. 135-142, 2010.

(C) Springer-Verlag Berlin Heidelberg 2010 
was defined discretely as $\|x\|_{T V}=\sum_{i} \sum_{j}\left(\left(\nabla_{1} x_{i j}\right)^{2}+\left(\nabla_{2} x_{i j}\right)^{2}\right)$ where $\nabla_{1}$ and $\nabla_{2}$ denote the forward finite difference operators on the first and second coordinates, respectively. Since both $L 1$ and TV norm regularization terms are nonsmooth, this problem is very difficult to solve. The conjugate gradient (CG) [3] and PDE [4] methods were used to attack it. However, they are very slow and impractical for real MR images. Computation became the bottleneck that prevented this good model (1) from being used in practical MR image reconstruction.

Other methods tried to reconstruct compressed MR images by performing $L_{p}$-quasinorm $(p<1)$ regularization optimization [5] 6] 7]. These nonconvex methods do not always give global minima and are also relatively slow. Trzasko et al. 8] used the homotopic nonconvex $L_{0}$-minimization to reconstruct MR images. It was faster than those $L_{p}$-quasinorm regularization methods. However, it still needed $1-3$ minutes to obtain reconstructions of $256 \times 256$ images in MATLAB on a $3 \mathrm{GHz}$ desktop computer. Recently, two fast methods were proposed to directly solve (1). In 9, Ma et al. proposed an operator-splitting algorithm (TVCMRI) to solve the MR image reconstruction problem. In [10, a variable splitting method (RecPF) was proposed to solve the MR image reconstruction problem. Both of them can replace iterative linear solvers with Fourier domain computations, which can gain substantial time savings. In MATLAB on a 3 $\mathrm{GHz}$ desktop computer, they can be used to obtain good reconstructions of $256 \times 256$ images in ten seconds or less. They are two of the fastest MR image reconstruction methods so far.

In this paper, we propose a new MR image reconstruction method based on the combination of variable and operator splitting techniques. We decompose the hard composite regularization problem (11) into two simpler regularization subproblems by: 1) splitting variable $x$ into two variables $\left\{x_{i}\right\}_{i=1,2} ; 2$ ) performing operator splitting to minimize total variation regularization and L1 norm regularization subproblems over $\left\{x_{i}\right\}_{i=1,2}$ respectively and 3) obtaining the solution $x$ by linear combination of $\left\{x_{i}\right\}_{i=1,2}$. This includes both variable splitting and operator splitting. We call it the Composite Splitting Algorithm (CSA). Motivated by the effective acceleration scheme in Fast Iterative Shrinkage-Thresholding Algorithm (FISTA) 11, the proposed CSA is further accelerated with an additional acceleration step. Numerous experiments have been conducted on real MR images to compare the proposed algorithm with previous methods. Experimental results show that it impressively outperforms previous methods for the MR image reconstruction in terms of both reconstruction accuracy and computation complexity.

\section{Related Acceleration Algorithm}

In this section, we briefly review the FISTA in [11, since our methods are motivated by it. FISTA consider to minimize the following problem:

$$
\min \left\{F(x) \equiv f(x)+g(x), x \in \mathbf{R}^{\mathbf{n}}\right\}
$$

where $f$ is a smooth convex function with Lipschitz constant $L_{f}$, and $g$ is a convex function which may be nonsmooth. 

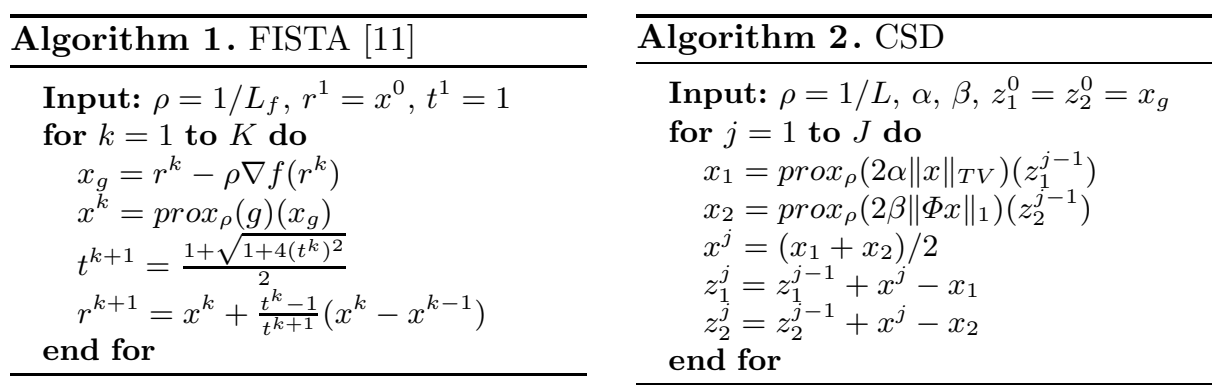

$\epsilon$-optimal Solution: Suppose $x^{*}$ is an optimal solution to (2). $x \in \mathbf{R}^{p}$ is called an $\epsilon$-optimal solution to (2) if $F(x)-F\left(x^{*}\right) \leq \epsilon$ holds.

Gradient: $\nabla f(x)$ denotes the gradient of the function $f$ at the point $x$.

The proximal map: given a continuous convex function $g(x)$ and any scalar $\rho>0$, the proximal map associated to function $g$ is defined as follows [11] [12]:

$$
\operatorname{prox}_{\rho}(g)(x):=\arg \min _{u}\left\{g(u)+\frac{1}{2 \rho}\|u-x\|^{2}\right\}
$$

Algorithm 1 outlines the FISTA. It can obtain an $\epsilon$-optimal solution in $\mathcal{O}(1 / \sqrt{\epsilon})$ iterations.

Theorem 1. (Theorem 4.1 in [11]): Suppose $\left\{x^{k}\right\}$ and $\left\{r^{k}\right\}$ are iteratively obtained by the FISTA, then, we have

$$
F\left(x^{k}\right)-F\left(x^{*}\right) \leq \frac{2 L_{f}\left\|x^{0}-x^{*}\right\|^{2}}{(k+1)^{2}}, \forall x^{*} \in X_{*}
$$

The efficiency of the FISTA highly depends on being able to quickly solve its second step $x^{k}=\operatorname{prox}_{\rho}(g)\left(x_{g}\right)$. For simpler regularization problems, it is possible, i.e, the FISTA can rapidly solve the $L 1$ regularization problem with $\operatorname{cost} \mathcal{O}(n \log (n))$ [1] (where $n$ is the dimension of $x$ ), since the second step $x^{k}=\operatorname{prox}_{\rho}\left(\beta\|\Phi x\|_{1}\right)\left(x_{g}\right)$ has a close form solution; It can also quickly solve the TV regularization problem, since the step $x^{k}=\operatorname{prox}_{\rho}\left(\alpha\|x\|_{T V}\right)\left(x_{g}\right)$ can be compute with cost $\mathcal{O}(n)$ [12. However, the FISTA can not efficiently solve the composite $L 1$ and TV regularization problem (1), since no efficient algorithm exists to solve the step

$$
x^{k}=\operatorname{prox}_{\rho}\left(\alpha\|x\|_{T V}+\beta\|\Phi x\|_{1}\right)\left(x_{g}\right) .
$$

To solve the problem (11), the key problem is thus to develop an efficient algorithm to solve problem (4). In the following section, we will show that a scheme based on composite splitting techniques can be used to do this.

\section{Algorithm}

From the above introduction, we know that, if we can develop a fast algorithm to solve problem (4), the MR image reconstruction problem can then be efficiently 
solved by the FISTA, which obtains an $\epsilon$-optimal solution in $\mathcal{O}(1 / \sqrt{\epsilon})$ iterations. Actually, problem (4) can be considered as a denoising problem:

$$
x^{k}=\arg \min _{x}\left\{\frac{1}{2}\left\|x-x_{g}\right\|^{2}+\rho \alpha\|x\|_{T V}+\rho \beta\|\Phi x\|_{1}\right\}
$$

We use composite splitting techniques to solve this problem: 1) splitting variable $x$ into two variables $\left.\left\{x_{i}\right\}_{i=1,2} ; 2\right)$ performing operator splitting over each of $\left\{x_{i}\right\}_{i=1,2}$ independently and 3 ) obtaining the solution $x$ by linear combination of $\left\{x_{i}\right\}_{i=1,2}$. We call it Composite Splitting Denoising (CSD) method, which is outlined in Algorithm 2, Its validity is guaranteed by the following theorem:

Theorem 2. Suppose $\left\{x^{j}\right\}$ the sequence generated by the CSD. Then, $x^{j}$ will converge to $\operatorname{prox}_{\rho}\left(\alpha\|x\|_{T V}+\beta\|\Phi x\|_{1}\right)\left(x_{g}\right)$, which means that we have $x^{j} \rightarrow$ $\operatorname{prox}_{\rho}\left(\alpha\|x\|_{T V}+\beta\|\Phi x\|_{1}\right)\left(x_{g}\right)$.

Due to page limitations, the proof for this theorem is given in the supplemental material.

Combining the CSD with FISTA, a new algorithm, FCSA, is proposed for MR image reconstruction problem (1). In practice, we found that a small iteration number $J$ in the CSD is enough for the FCSA to obtain good reconstruction results. Especially, it is set as 1 in our algorithm. Numerous experimental results in the next section will show that it is good enough for real MR image reconstruction.

Algorithm 4 outlines the proposed FCSA. In this algorithm, if we remove the acceleration step by setting $t^{k+1} \equiv 1$ in each iteration, we will obtain the Composite Splitting Algorithm (CSA), which is outlined in Algorithm 3, A key feature of the FCSA is its fast convergence performance borrowed from the FISTA. From Theorem 1, we know that the FISTA can obtain an $\epsilon$-optimal solution in $\mathcal{O}(1 / \sqrt{\epsilon})$ iterations.

Another key feature of the FCSA is that the cost of each iteration is $\mathcal{O}(n$ $\log (n))$, as confirmed by the following observations. The step 4, 6 and 7 only involve adding vectors or scalars, thus cost only $\mathcal{O}(n)$ or $\mathcal{O}(1)$. In step $1, \nabla f\left(r^{k}=\right.$ $R^{T}\left(R r^{k}-b\right)$ since $f\left(r^{k}\right)=\frac{1}{2}\left\|R r^{k}-b\right\|^{2}$ in this case. Thus, this step only costs $\mathcal{O}(n \log (n))$. As introduced above, the step $x^{k}=\operatorname{prox}_{\rho}\left(2 \alpha\|x\|_{T V}\right)\left(x_{g}\right)$ can be computed quickly with cost $\mathcal{O}(n)$ [12]; The step $x^{k}=\operatorname{prox}_{\rho}\left(2 \beta\|\Phi x\|_{1}\right)\left(x_{g}\right)$ has a close form solution and can be computed with cost $\mathcal{O}(n \log (n))$. In the step $x^{k}=\operatorname{project}\left(x^{k},[l, u]\right)$, the function $x=\operatorname{project}(x,[l, u])$ is defined as: 1) $x=x$ if $l \leq x \leq u$;2) $x=l$ if $x<u$; and 3) $x=u$ if $x>u$, where $[l, u]$ is the range of $x$. For example, in the case of MR image reconstruction, we can let $l=0$ and $u=255$ for 8 -bit gray MR images. This step costs $\mathcal{O}(n)$. Thus, the total cost of each iteration in the FCSA is $\mathcal{O}(n \log (n))$.

With these two key features, the FCSA efficiently solves the MR image reconstruction problem (11) and obtains better reconstruction results in terms of both the reconstruction accuracy and computation complexity. The experimental results in the next section demonstrate its superior performance compared with all previous methods for compressed MR image reconstruction. 


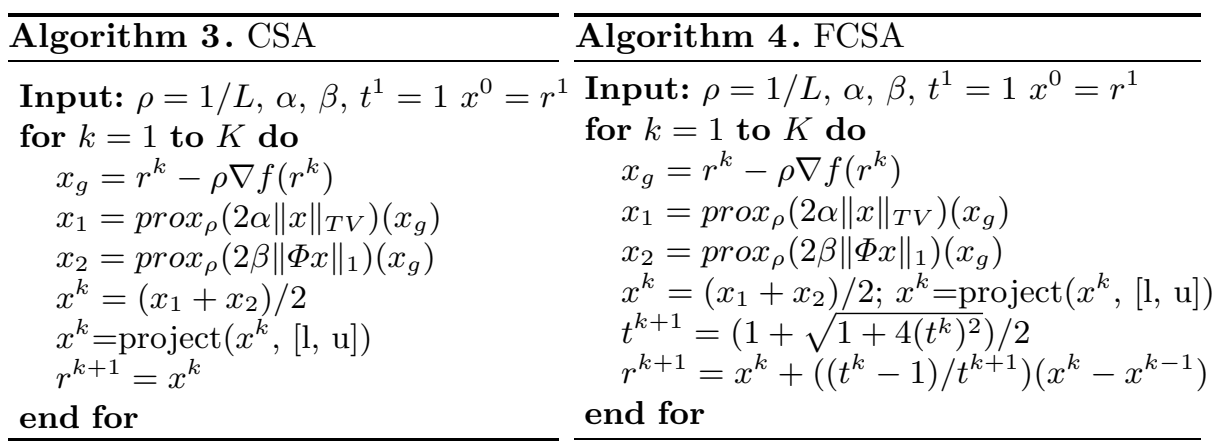

\section{Experiments}

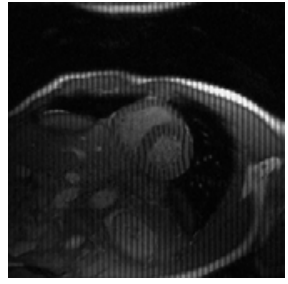

(a)

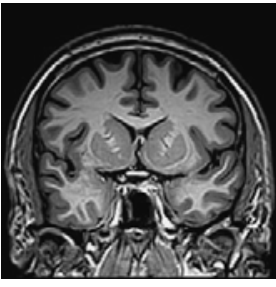

(b)

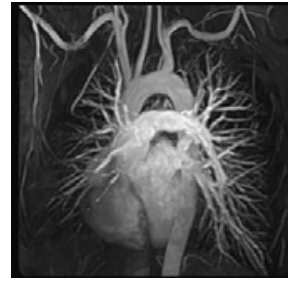

(c)

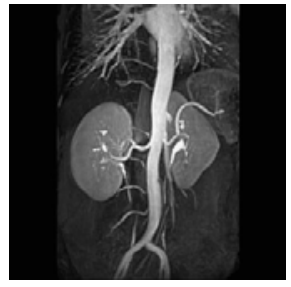

(d)

Fig. 1. MR images: a) Cardiac; (b) Brain; (C) Chest and (d) Artery

\subsection{Experiment Setup}

Suppose a MR image $x$ has $n$ pixels, the partial Fourier transform $R$ in problem (11) consists of $m$ rows of a $n \times n$ matrix corresponding to the full $2 \mathrm{D}$ discrete Fourier transform. The $m$ selected rows correspond to the acquired $b$. The sampling ratio is defined as $m / n$. The scanning duration is shorter if the sampling ratio is smaller. In MR imaging, we have certain freedom to select the rows, which correspond to certain frequencies. In the k-space, we randomly obtain more samples in low frequencies and less samples in higher frequencies. This sampling scheme is the same of those in [3] 9, 10] and has been widely used for compressed MR image reconstruction. Following these guidelines, we randomly created sampling matrices. Practically, the sampling scheme and speed in MR imaging also depend on the physical and physiological limitations [3].

We implement our CSA and FCSA for problem (11) and apply them on 2D real MR images. All experiments are conducted on a $2.4 \mathrm{GHz} \mathrm{PC}$ in Matlab environment. We compare the CSA and FCSA with the classic MR image reconstruction method based on the CG [3. We also compare them with two of the fastest MR image reconstruction methods, TVCMRI [9] and RecPF [10]. For fair comparisons, we download the codes from their websites and carefully follow 
their experiment setup. For example, the observation measurement $b$ is synthesized as $b=R x+\mathbf{n}$, where $\mathbf{n}$ is Gaussian white noise with standard deviation $\sigma=0.01$. The regularization parameter $\alpha$ and $\beta$ are set as 0.001 and $0.035 . R$ and $b$ are given as inputs, and $x$ is the unknown target. For quantitative evaluation, we compute the Signal-to-Noise Ratio (SNR) for each reconstruction result.

\subsection{Numerical Results}

We apply all methods on four 2D MR images: cardiac, brain, chest and artery respectively. Figure 1 shows these images. For convenience, they have the same size of $256 \times 256$. The sample ratio is set to be approximately $25 \%$. To perform fair comparisons, all methods run 50 iterations except that the CG runs only 8 iterations due to its higher complexity. The CPU time and SNR are traced in each iteration for each of methods. To reduce the randomness, we run each experiments 100 times for each parameter setting of each method.

Figure 2 shows the visual comparisons of the reconstructed results by different methods. Figure 3 gives the performance comparisons between different methods in terms of the CPU time over SNR.The FCSA always obtains the best reconstruction results on all MR images by achieving the highest SNR in less CPU time. The CSA is always inferior to the FCSA, which shows the effectiveness of acceleration steps in the FCSA for the MR image reconstruction. While the classical CG 3] is far worse than others because of its higher cost in each iteration, the RecPF sound be slightly better than the TVCMRI, which is consistent to the observations in [9] and [10].

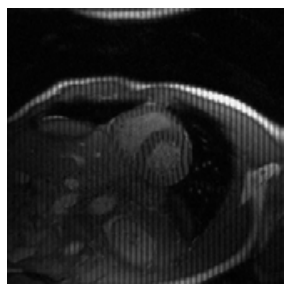

(a)

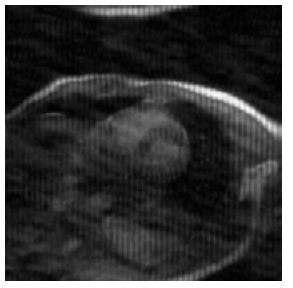

(b)

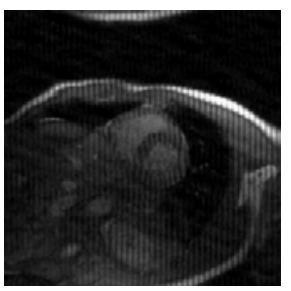

(e)

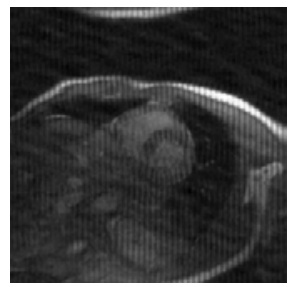

(c)

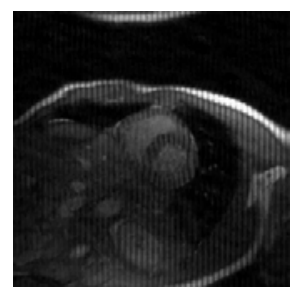

(f)

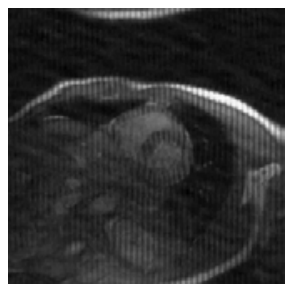

(d)

Fig. 2. Cardiac MR image reconstruction (a) Original image; (b), (c), (d) (e) and (f) are the reconstructed images by the CG [3], TVCMRI 9], RecPF 10], CSA and FCSA. Their SNR are 9.86, 14.43, 15.20, 16.46 and $17.57(\mathrm{db})$. Their CPU time are 2.87, 3.14, $3.07,2.22$ and $2.29(\mathrm{~s})$. 


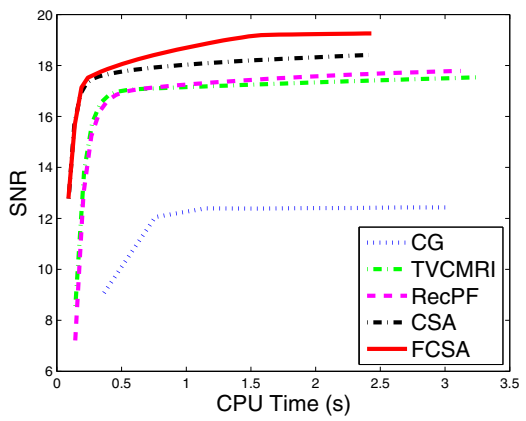

(a)

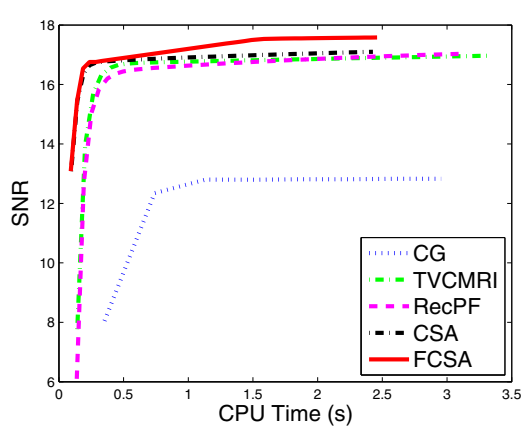

(c)

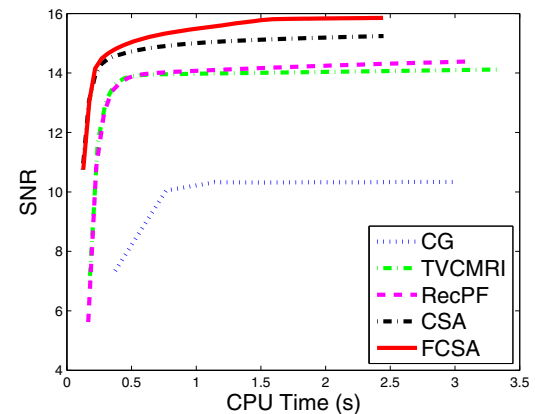

(b)

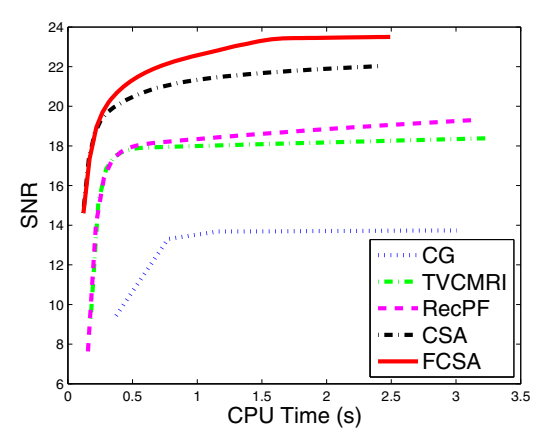

(d)

Fig. 3. Performance comparisons (CPU-Time vs. SNR) on different MR images: a) Cardiac image; (b) Brain image; (C) Chest image and (d) Artery image

The reconstructed results produced by the FCSA are far better than those produced by the CG, TVCMRI and RecPF. The reconstruction performance of the FCSA is always the best in terms of both the reconstruction accuracy and the computational complexity, which clearly demonstrate the effective and efficiency of the FCSA for the compressed MR image construction.

\section{Conclusion}

We have proposed an efficient algorithm for the compressed MR image reconstruction. Our work has the following contributions. First, the proposed FCSA can efficiently solve a composite regularization problem including both TV term and L1 norm term, which can be easily extended to other medical image applications. Second, the computational complexity of the FCSA is only $\mathcal{O}(n \log (n))$ in each iteration. It also has strong convergence properties. These properties make the real compressed MR image reconstruction much more feasible than before. Finally, we conduct numerous experiments to compare different reconstruction methods. Our method is shown to impressively outperform the classic methods and two of fastest methods so far in terms of both accuracy and complexity. 


\section{References}

1. Candes, E.J., Romberg, J., Tao, T.: Robust uncertainty principles: Exact signal reconstruction from highly incomplete frequency information. IEEE Transactions on Information Theory 52, 489-509 (2006)

2. Donoho, D.: Compressed sensing. IEEE Transactions on Information Theory 52(4), 1289-1306 (2006)

3. Lustig, M., Donoho, D., Pauly, J.: Sparse mri: The application of compressed sensing for rapid mr imaging. Magnetic Resonance in Medicine 58, 1182-1195 (2007)

4. He, L., Chang, T.C., Osher, S., Fang, T., Speier, P.: Mr image reconstruction by using the iterative refinement method and nonlinear inverse scale space methods. Technical report, UCLA CAM 06-35 (2006)

5. Ye, J., Tak, S., Han, Y., Park, H.: Projection reconstruction mr imaging using focuss. Magnetic Resonance in Medicine 57, 764-775 (2007)

6. Chartrand, R.: Exact reconstruction of sparse signals via nonconvex minimization. IEEE Signal Processing Letters 14, 707-710 (2007)

7. Chartrand, R.: Fast algorithms for nonconvex compressive sensing: Mri reconstruction from very few data. In: Proceedings of ISBI (2009)

8. Trzasko, J., Manduca, A., Borisch, E.: Highly undersampled magnetic resonance image reconstruction via homotopic $l 0$-minimization. IEEE Transactions on Medical Imaging 28, 106-121 (2009)

9. Ma, S., Yin, W., Zhang, Y., Chakraborty, A.: An efficient algorithm for compressed $\mathrm{mr}$ imaging using total variation and wavelets. In: Proceedings of CVPR (2008)

10. Yang, J., Zhang, Y., Yin, W.: A fast alternating direction method for tvl1-l2 signal reconstruction from partial fourier data. IEEE Journal of Selected Topics in Signal Processing, Special Issue on Compressive Sensing 4(2) (2010)

11. Beck, A., Teboulle, M.: A fast iterative shrinkage-thresholding algorithm for linear inverse problems. SIAM Journal on Imaging Sciences 2(1), 183-202 (2009)

12. Beck, A., Teboulle, M.: Fast gradient-based algorithms for constrained total variation image denoising and deblurring problems. IEEE Transaction on Image Processing 18(113), 2419-2434 (2009) 